\title{
Time-Frequency Analysis of Cabin Noise Using EEMD-ICA Approaches
}

\author{
Wang Li, Zhou Yi-qi, Yu Gang and Mi Yong-zhen \\ Key Laboratory of High Efficiency and Clean Mechanical Manufacture, Ministry of \\ Education, School of Mechanical Engineering Shandong University \\ lilywang460@foxmail.com
}

\begin{abstract}
The interior noise of construction machinery is complex non-stationary signal caused by a variety of excitation source; the noise source must be determined to minimize its radiation. There are, however, considerably fewer techniques available if only a single-channel measurement is available and yet single-channel measurements are important where minimal instrumentation complexity is required. The combined techniques of empirical mode decomposition (EEMD) and the improvement of independent component analysis (ICA) were applied to analysis the parotic noise signals of bulldozer cabin in our study. According to the correlated measurement standard, the combining method of experiment and analysis for the cabin interior and outside noise and vibration signals was applied to research the noise source effectively. With the experiment and coherence analysis for the cabin interior and outside noise and vibration signals, the techniques of EEMD-ICA were valid on the noise source separation and identification. Taking into account the machine running characteristics and the layout of machine structures, and based on the frequency characteristics of the noise and vibration signals, we concluded that the main sources of indoor noise was combustion noise of diesel engine and mechanical radiation noise of cabin parts caused by the diesel engine vibration transmission. Generation of noise sources, transmission paths of air-borne or structure-borne noises were well researched in order to control the interior noise. Finally, some modifications were taken for different noise sources and the noise level was reduced to a satisfied level.
\end{abstract}

Keywords: cab interior noise; construction machinery; EEMD; ICA; time-frequency analysis

\section{Introduction}

The rapid development of Chinese city construction, the tearing down and building of city, and the construction of basic infrastructures, directly bring the great increase amount of construction machinery applied to more projects. The engine of machinery discharges noise with high level during working, for example, the inlet noise and exhaust noise, noise radiated from the body, and the noise from gears. As an important indicator of NVH (Noise, Vibration and Harshness), cabin noise in construction machinery raised more and more common concerns of consumers and manufacturers. About the engineering machinery cabin noise forecast, measuring evaluation and control methods, there were a large amount of literatures on theoretical and experimental research [1-3].

We take a certain type of crawler dozer as an example, the upper limit sound level value of parotic noise between new and old national standard of China for the dozer is over $5 \mathrm{~dB}$ [4-5]. 
It means that not only basic parts for construction machinery and other key parts manufacturers have to reduce noise power of diesel engines, transmissions and hydraulic components and other equipment, but also the assembly plants would to promote comprehensive technology of vibration damping and noise reducing.

The main components of a large bulldozer are distributed in different location, and the main noise sources and vibration excitations have different influence on the bulldozers. The noise sources in the time domain and frequency domain have respective periods of time and frequency characteristics.

In the case of several noise sources coexistence, we should try to reduce the highest source of noise in order to achieve significant noise reduction effect under the principle of superposition of noise. In order to achieve the goal, the primary task is to determine the noise source. Because multiple noise sources work simultaneously, the resulting noise signals test collected contain a variety of complex transient non-stationary signals and a few stationary signals superimposed. It is difficult to reveal the complex noise and vibration phenomena using a single method of analysis [6-7]. So, the measurement and analysis of the cabin noise becomes a very important step for reducing noise, protecting the environment and improving the complete machine performance.

In the research field of noise source identification, based on independent component analysis (ICA) of blind source separation (BSS) methods is the hot topic of research. Blind Sources Separation (BSS) is one of the multiple signals processing method. It is the process of recovering unknown independent source signals from sensor measurements which are unknown combinations of the source signals according to the statistical characteristic of input source signal to recovering the source signal independent component. As an effective technique, independent component analysis is closely related to BSS and has practical applications for solving this type of problem in biomedicine, speech separation, image processing, and other fields [8-10].

Using independent component analysis, there is an important restriction: the channel number must not be less than the number of source signals. But it is difficult to know the number of source signals in advance, and increase acquisition channel through infinite number of methods to identify the source signal will cause unnecessary waste and even increase the test difficulty.

Ensemble empirical mode decomposition (EEMD) is employed to decompose nonlinear and non-stationary signal into a series of adaptive linear, smooth intrinsic mode function (IMF) signals which meet the requirements of the blind source separation.

But this does not guarantee the independence of the intrinsic mode function. If we have adaptive decomposition of a single channel signal, and combination the IMFs into a new independent signal, thus solve the undetermined problem of blind source identification from single sampling signal. The combined techniques of EEMD-ICA have been applied in the study of some complex problems [11, 12], showing the advantages of this method.

The combined techniques of ensemble empirical mode decomposition (EEMD) and the improvement of independent component analysis (FICA) are applied to analysis the the parotic noise signals of bulldozer cabin in our study. And techniques of coherence analysis and time frequency analysis are used to identify the noise sources. By comparing the spectrum diagrams of main noise source signals separated, and calculating coherence functions of the near field vibration and noise measurement signals, we will differentiate and analysis cabin noise sources. 


\section{Method}

\subsection{Ensemble Empirical Mode Decomposition}

Empirical mode decomposition (EMD) is the core of Ensemble empirical mode decomposition (EEMD) method. EMD is a kind of adaptive and efficient signal decomposition method that decomposes any complicated time series into a finite set of oscillatory modes called IMFs, and obtain instantaneous frequency data. Although the EMD is a good signal decomposition approach, when the signal discontinued in a certain frequency component, this method is not suitable which results in modal aliasing phenomenon. EEMD is a more robust and noise-assisted version of the EMD algorithm [13].

The updated algorithm defines the IMF set for an ensemble of trials, each one obtained by applying EMD to the signal of interest with added independent, identically distributed white noise of the same standard deviation. The algorithm not only provides uniform distribution random signal for analysis, and soothes the pulse interference, can effectively inhibit the modal aliasing caused by abnormal disturbance. And multiple decomposition results are applied to lumped average calculation. Thus it is significantly reduce the chance of mode mixing and make the decomposed the IMF more meaningful. The procedure of the EEMD method can be briefly summarized as follows:

Step 1:Add white noise with a predefined noise amplitude to the signal to be analyzed.

Step 2:Use the EMD method to decompose the newly generated signal.

Step 3:Repeat the above signal decomposition with different white noise, in which the amplitude of the added white noise is fixed.

Step 4:Calculate the ensemble means of the decomposition results as final results.

Using this signal processing method, a multi-component signal $x(k)$, is decomposed into a finite number of intrinsic mode functions (IMFs) and a residue. In each decomposition process, the added white noise helps to perturb the analyzed signal and enables the EMD method to visit all possible solutions in the finite neighborhood of the true final IMF. Based on the property of zero mean of white noise, the added white noise can be canceled out in the final ensemble mean if there are sufficient trials. Only the signal itself can survive in the final decomposition results.

\subsection{Independent Component Analysis}

ICA is a blind source separation (BSS) technique in which recorded signals are separated into their independent constituent components or sources.

Under certain optimization rules the ICA technique is to separate instantaneously mixed signals from the channel matrix into the mutual statistical independence of the non-Gaussian Sources. There are a number of different algorithms currently available to determine this unmixing matrix including infomax, FastICA, FJADE and CICA. Among these algorithms, FastICA is an efficient and popular algorithm for independent component analysis invented by Aapo Hyvärinen [14].

The fast ICA algorithm was implemented for this paper due to its shorter computational time compared to the other ICA techniques. 


\subsection{Analysis Process}

The combined techniques process of the parotic noise signal of bulldozer cabin can be summarized in the following.

First EEMD algorithm is applied to calculate the IMFs of a given parotic noise time series. After a set of averaged IMFs derived, single channel parotic noise signal and its IMFs combination become the new multidimensional signals. We know that if there is some internal relationship between two random variables, then through the corresponding statistical methods, the characterization of real IMF components properties will have relative correlation with the original signal. Thus, we can use the correlation coefficients of IMFs and the parotic noise signal as a criterion to decide which IMFs should be retained and which IMFs should be eliminated [15]. According to the correlation coefficients value, we can estimate the main noise sources.

The FastICA algorithm is applied to the retained set of IMFs and provides us with mixing and unmixing matrices $\mathrm{M}$ and $\mathrm{W}$, and a matrix of ICs $S$ as an output, where the matrix of retained IMFs $(\boldsymbol{C})$ satisfies the equation $\boldsymbol{C}=\boldsymbol{M S}$, and $\boldsymbol{S}=\boldsymbol{W C}$. Thus the appearance of the desired source in the original signal is obtained.

Based on the characteristics of signal frequency domain analysis, combined with a bulldozer corresponding prior knowledge of each component, independent components of the original signal are identified, and the details of interior noise signal are researched.

\section{Cab Noise Sources Identification}

\subsection{Experiment Setup}

According to some correlated measurement standards [16], the experiment for the cabin interior and outside noise and vibration signals was applied to research the noise source for a certain type of bulldozers in stationary work cycle mode. The engine and hydraulic system was warmed to normal operating conditions as specified by the machine manufacturer. All liquid systems were filled within the range specified by the manufacturer. The engine rotational speed was set at the maximum value $2000 \mathrm{rpm}$ with no load, as specified by the machine manufacturer.

When engine rotational speed was stable, noise signal at ear height of the driver position in cabin was measured. In order to verify the correctness of noise source identification results, near-field noise and vibration related both inside and outside other parts of the bulldozer was measured. With reference to the vibration transfer path and mode of vibration accelerometers were decorated reference points. Table 1 showed the reference points in and out of the bulldozer.

Table 1. Reference Points in and out of the Bulldozer

\begin{tabular}{llll}
\hline 1 & Near the transfer case $10 \mathrm{~cm}$ & 2 & Air filter near $10 \mathrm{~cm}$ \\
\hline 3 & Left part of operation table & 4 & Right part of operation table \\
\hline 5 & Instrument panel surface & 6 & Middle part of cab ceiling \\
\hline 7 & Near the driver ear position & 8 & Left door surface \\
\hline 9 & Right door surface & 10 & Front part of cab floor \\
\hline
\end{tabular}

The time domain waveform of interior cab noise signal was shown in Figure 1. With the adaptive window function, short time Fourier transform analysis was applied to research time-frequency characteristics of cab noise signal as shown in Figure 2. 
With the analysis of time-frequency characteristics in Figure 2, we could notice that the frequencies corresponding to these big amplitude values are mainly concentrated within the $550 \mathrm{~Hz}$ in low frequency band. And noise signal at low frequency appears some fluctuation.

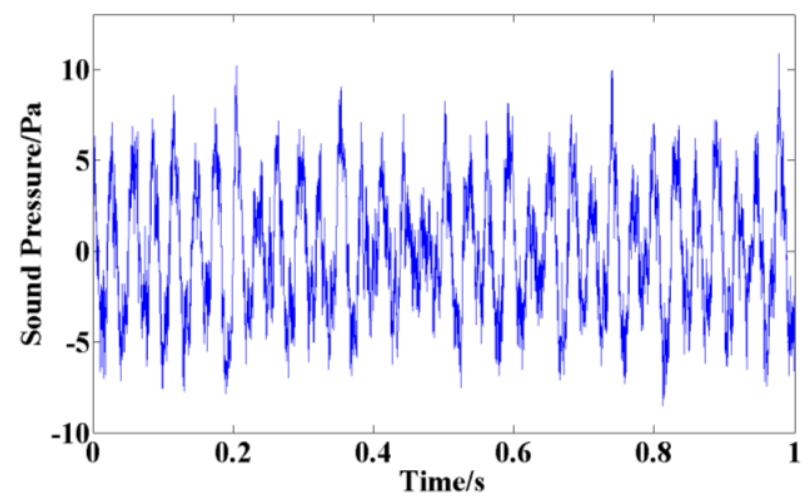

Figure 1. Cab Noise Signals in Time Domain

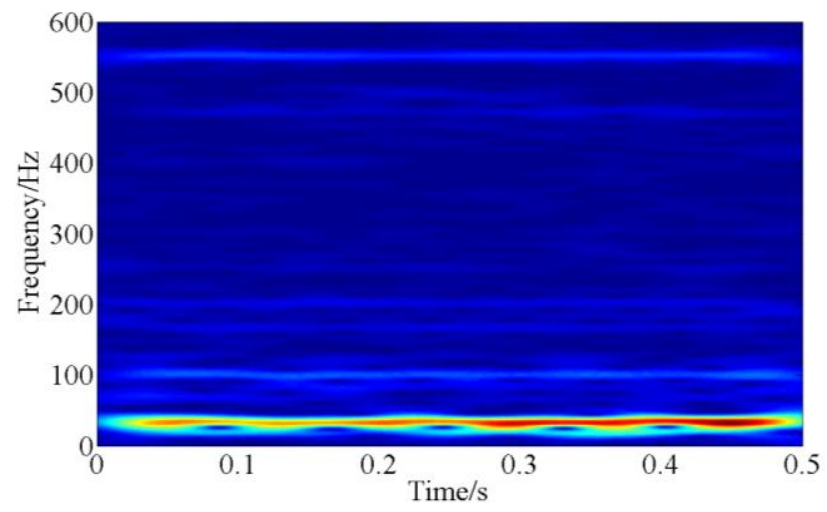

Figure 2. Time-frequency Characteristic of Cab Noise Signals

\subsection{Noise Source Separation}

After the pretreatment as remove the mean of data series, the ratio of the standard deviation of the added noise is set to 0.2 , and ensemble number for the EEMD was set to 400 times, the cab noise signals were decomposed. Then we got eight IMF components and an approximate residual. The result was shown in Figure 3.

Figure 3 illustrated the EEMD decompositions of the mixed cab noise. The IMFs extracted from original signals were oscillatory functions with varying amplitude and frequency. RST was a residual component, which contained negligible energy. From Figure 4, we could directly see that the IMF1 IMF4 obviously were constituted by the high frequency signals, IMF4 IMF8 were constituted by the middle or low frequency signals and the frequency of each IMF component composition was relatively single. The IMF4 IMF6 components had bigger amplitude than other IMFs. It represented that the energy of the components were larger in the original signal. IMF7 IMF11 contained little signal energy, so the number of IMFs needed to be reduced. 


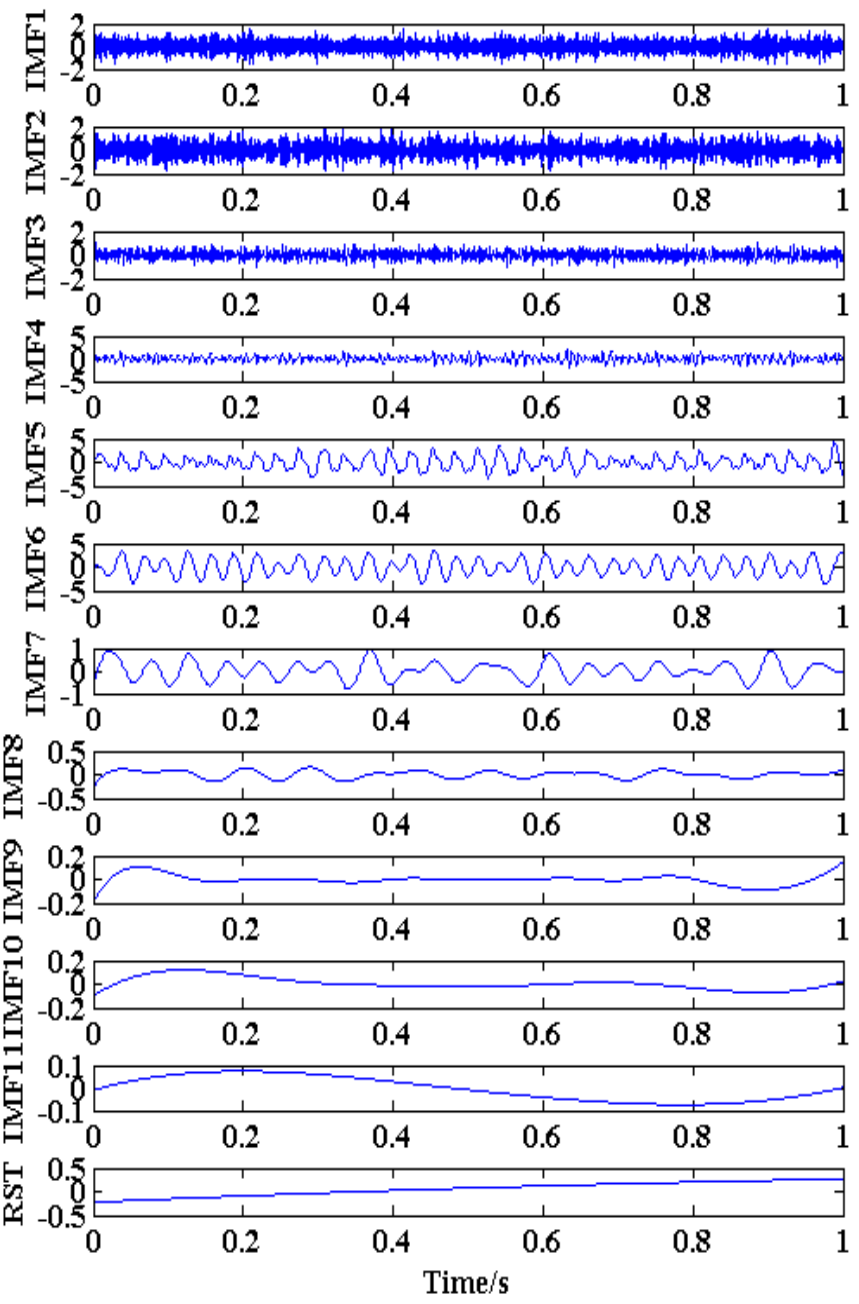

Figure 3. Decomposition Results of EEMD for cab noise signals

Table 2. Coefficient between IMFs and the source signals

\begin{tabular}{llllll}
\hline IMF1 & IMF2 & IMF3 & IMF4 & IMF5 & IMF6 \\
\hline 0.1973 & 0.2604 & 0.2661 & 0.3665 & 0.8686 & 0.8662 \\
\hline IMF7 & IMF8 & IMF9 & IMF10 & IMF11 & \\
\hline 0.2592 & 0.0586 & 0.0283 & $-1.4489 \mathrm{e}-4$ & -0.0030 & \\
\hline
\end{tabular}

Next, we used the the correlation coefficients of IMFs and the parotic noise signal to extract the main energy of all IMFs before IMFs were processed by the ICA.

The specific correlation coefficients calculation results were shown in Table 2, of which the correlation coefficients of IMF5, IMF6 were the largest, the correlation coefficients of IMF4 was bigger also, they retained the main energy of the original signal. Given the correlation coefficients of IMFs, we could estimate that the cab noise signals are mixed with three noise signal. 
After that FICA was applied to the leaved signals and thereby separated the original source signals as displayed in Figure 4. The independent components of the original signal were some different with IMF4 IMF6 which have varying amplitude and frequency.
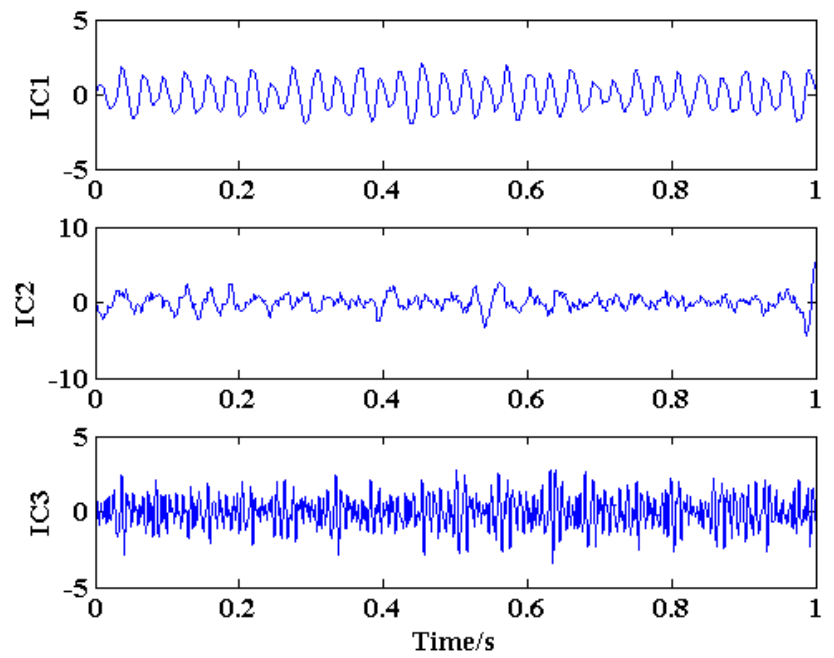

Figure 4. Separated Results of ICA for Cab Noise Signals

\section{Results and Discussion}

With the adaptive window function, short time Fourier transform analysis was applied to research time-frequency characteristics of ICs as shown in Figures 5 7. It is clear that all the ICs are constituted by the middle or low frequency signals. And the frequency is not stable in time domain but is fluctuated in a certain range.

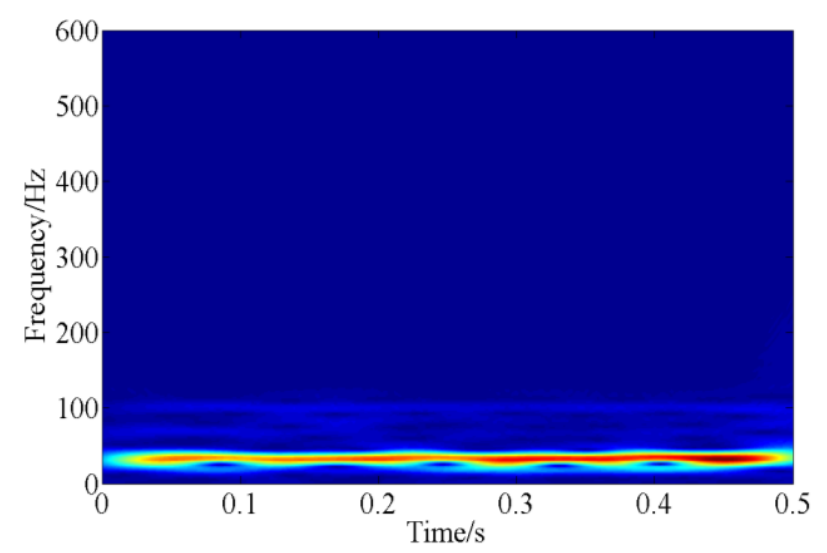

Figure 5. Time-frequency characteristic of IC1 


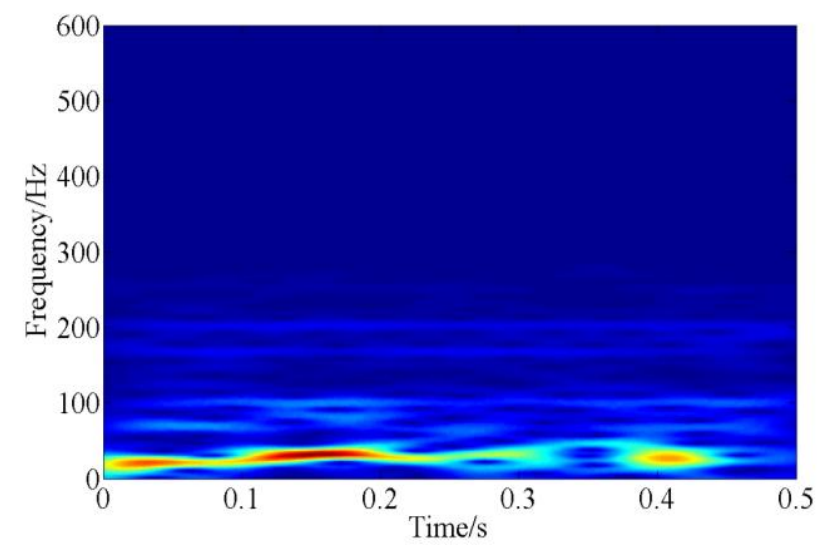

Figure 6. Time-frequency characteristic of IC2

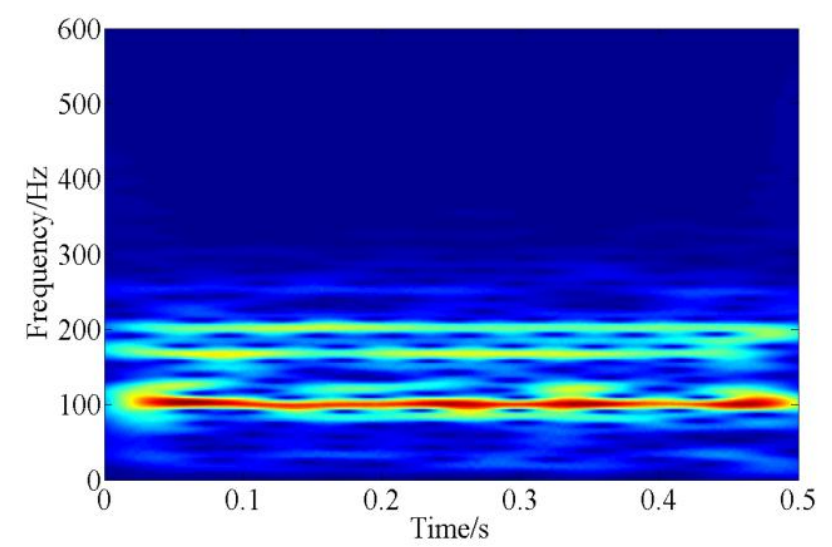

Figure 7. Time-frequency characteristic of IC3

The bulldozer cab made up by many parts, and structural modal parameter of these parts may not be the same. When the vibration excitation of engine transmits into the cab, and some certain structural modal frequency corresponding interior parts of the cab is consistent with the vibration frequency, the components in cab would vibrate and radiate noise. The most outstanding frequency of IC1 (Figure 5) is around $33.3 \mathrm{~Hz}$. The main frequency components is consistent with the 1 order of this diesel engine rotational frequency $33.3 \mathrm{~Hz}$ $(2000 / 60=33.3 \mathrm{~Hz})$. Therefore IC1 is likely to be caused by the vibration of engine components radiated noise.

The main frequency of IC2 (Figure 6) is around $16.7 \mathrm{~Hz}$. By the 1 order of the diesel engine rotational frequency $33.3 \mathrm{~Hz}(2000 / 60=33.3 \mathrm{~Hz})$, the IC2 main frequency is correspond to the rotation frequency of the $1 / 2$ order, which matched with the fundamental vibration frequency of four stroke engines. And the frequency of IC2 is not stable in time domain especially after $0.3 \mathrm{~s}$. With the time-frequency analysis method we can research the no stationary periodic signal in details.

As shown in Figure 7, the energy distribute of IC3 is complicated, mainly concentrated within $300 \mathrm{~Hz}$. And the most outstanding frequency is about $100 \mathrm{~Hz}, 200 \mathrm{~Hz}$. Our bulldozer engine is four stroke diesel engine with six cylinders, and the ignition frequency calculation formula is: $f=n \cdot z /(60 \cdot \tau), n$ - engine speed, $\mathrm{r} / \mathrm{min} ; z$ - number of cylinders; $\tau$ - stroke number, value is set 2 for four stroke. At the diesel engine rotating speed of $2000 \mathrm{r} / \mathrm{min}$, the ignition frequency is $100 \mathrm{~Hz}$. The main frequency component of IC3 is just corresponding to three 
times of the 1 order engine rotational frequency, which is the same as the ignition frequency. So we can conclude that the IC3 is likely caused by the engine combustion noise radiation.

In order to verify the correctness of noise source identification results, the coherence analysis of IC1 respectively with the vibration acceleration signal of various parts and components in the cab near was applied in frequency domain. The coherence functions were calculated with IC1 and the vibration or noise test signals at frequency $33.3 \mathrm{~Hz}$. The specific coherence functions calculation results were shown in Table 3. It is clear that IC1, all vibration signals and noise near the transfer case have the large coherence functions, while noise near air filter has the small coherence function. Based on the analysis, component IC1 may come from the vibration radiation noise of many different parts. And the 1 order of this diesel engine rotational frequency $33.3 \mathrm{~Hz}$ is very important to noise treatment for this bulldozer cab. So some methods could be applied to deal with the cab noise problems, for instance, structural modal frequency planning of various components for avoiding resonance, to improve the connection of various parts, to improve the shock absorber design, and so on.

Table 3. Coherence Analysis between IC1 and the Test Signals

\begin{tabular}{llll}
\hline Left part of operation table & 0.9634 & Front part of cab floor & 0.9392 \\
\hline Right part of operation table & 0.9729 & Left door surface & 0.9103 \\
\hline Instrument panel surface & 0.9312 & Right door surface & 0.9566 \\
\hline Middle part of cab ceiling & 0.9520 & Noise near the transfer case & 0.8692 \\
\hline Noise near the air filter & 0.5526 & & \\
\hline
\end{tabular}

Taking into account the machine running characteristics and the layout of machine structures, and based on the frequency characteristics of the noise and vibration signals, we could make sure that the main source of cab noise is combustion noise of diesel engine and mechanical radiation noise of cabin parts caused by the diesel engine vibration transmission.

According to the comprehensive test and the analysis, some modifications were taken for different noise sources, for example, adapting the vibration system to improve the effect of vibration isolation, adding sound insulation materials to reduce incoming air noise, improving design of the cab floor to increase the structural stiffness to avoid the key resonance frequency, changing the operation table connected installation to improve vibration isolation, and so on. Specific adjustment and improvement measures were divided into two phases and the test results were shown in Table 4. Cab noise decreases at different engine rotational speed. After a preliminary improvement, the noise level is reduced to a satisfied level. Base on the generation of noise sources, transmission paths of air-borne or structure-borne noise, the test verifies the effectiveness of the adjustment and improvement measures.

Table 4. Cab Noise SPL Comparison before and after Improvement

\begin{tabular}{llll}
\hline \multirow{2}{*}{$\begin{array}{c}\text { Speed } \\
\text { /rpm }\end{array}$} & Original & $\begin{array}{c}\text { Take Sound } \\
\text { insulation measures }\end{array}$ & $\begin{array}{c}\text { Take } \\
\text { Integrated measures }\end{array}$ \\
\cline { 2 - 4 } & & 79.0 & 78.1 \\
\hline 1000 & 83.4 & 88.3 & 82.9 \\
\hline 1600 & 89.5 & 80.5 \\
\hline 2000 & 91.4 & 90.0 & $88.5(\mathrm{~A})$ \\
\hline
\end{tabular}




\section{Conclusion}

In this paper, the combined techniques of EEMD-ICA was applied to analysis the the parotic noise signals of bulldozer cabin. After the cab noise signals were decomposed and the correlation coefficients of IMFs and the parotic noise signal was used as a criterion to estimate the main noise sources. And the time frequency analysis was effectively used to identify the noise sources. With the combined method of experiment and coherence analysis for the cabin interior and outside noise and vibration signals, we verify that the techniques of EEMD-ICA is valid on the noise source separation and identification. Taking into account the machine running characteristics and the layout of machine structures, and based on the frequency characteristics of the noise and vibration signals, we can conclude that the main sources of cab noise are combustion noise of diesel engine and mechanical radiation noise of cabin parts caused by the diesel engine vibration transmission in the middle or low frequency. Generation of noise sources, transmission paths of air-borne or structure-borne noises were well analyzed in order to control the interior noise. Finally, some modifications were taken for different noise sources and the noise level was reduced to a satisfied level, the NVH performance of this bulldozer was greatly improved.

\section{References}

[1] W. Nakkula Jr, R. Zimmerli and R. Borough, "An example of noise control treatment for construction Machinery Cab Interiors", Earthmoving Industry Conference, (1981) April 6-8; Peoria, IL, United states.

[2] R. N. Gujarathi, K. S. Kolte, K. G. Kshirsagar, T. S. Salunke and M. D. Rao, "Panel contribution study of a commercial excavator cab using sound intensity measurements", 22nd National Conference on Noise Control Engineering, NOISE-CON 2007, (2007) October 22-24; Reno, NV, United states, pp. 1744-1751.

[3] C. R. Hartley and J. Choi, "Finite Element Overlay Technique for Predicting the Payne Effect in a FilledRubber Cab Mount", SAE International Journal of Passenger Cars - Mechanical Systems, vol. 5, no. 1, (2012), pp. 413-424.

[4] National standards in People's Republic of China, Earth-moving machinery-Noise Limits (China, GB16710.1-1996).

[5] National standards in People's Republic of China, Earth-moving machinery-Noise Limits (China, GB167102010).

[6] J. Chang, M. Kim and K. Min, "Detection of misfire and knock in spark ignition engines by wavelet transform of engine block vibration signals", Measurement Science and Technology, vol. 13, no. 7, (2002), pp. 1108-1114.

[7] R. Johnsson, "Cylinder pressure reconstruction based on complex radial basis function networks from vibration and speed signals", Mechanical Systems and Signal Processing, vol. 20, no. 8, (2006), pp. 19231940.

[8] A. Widodo, B. Yang and T. Han, "Combination of independent component analysis and support vector machines for intelligent faults diagnosis of induction motors", Expert Systems with Applications, vol. 32, no. 2, (2007), pp. 299-312.

[9] G. R. Naik, D. K. Kumar and H. Weghorn, "Performance comparison of ICA algorithms for isometric hand gesture identification using surface EMG”, 2007 International Conference on Intelligent Sensors, Sensor Networks and Information Processing, ISSNIP, (2007) December 3-6; Melbourne, VIC, Australia, pp. 613618.

[10] L. Yin, W. Fuxiang, L. Xiaoyan and Z. Jun, “An ICA-based image fusion scheme using only source images”, 2nd International Conference of Electrical and Electronics Engineering, ICEEE 2011, (2011) December 1-2; Macau, China, pp.589-596.

[11] K. T. Sweeney, S. F. McLoone and T. E. Ward, Tomas, "The use of ensemble empirical mode decomposition with canonical correlation analysis as a novel artifact removal technique", IEEE Transactions on Biomedical Engineering, vol. 60, no. 1, (2013), pp. 97-105.

[12] G. Yina, H. Shuhua and L. Yongtang, "Single-mixture source separation using dimensionality reduction of ensemble empirical mode decomposition and independent component analysis", Circuits, Systems, and Signal Processing, vol. 31, no. 6, (2012), pp. 2047-2060. 
[13] Z. H. Wu and N. E. Huang, "Ensemble empirical mode de-composition: a noise-assisted data analysis method", Advances in Adaptive Data Analysis, Theory and Applications, vol. 1, no. 1, (2009), pp. 1-41.

[14] A. Hyvarinen, "Fast and robust fixed-point algorithm for independent component analysis", IEEE Transactions on Neural Network, vol. 10, no. 3, (1999), pp. 626-634.

[15] J. Benesty, C. Jingdong and H. Yiteng, "On the importance of the pearson correlation coefficient in noise reduction”, IEEE Transactions on Audio, Speech and Language Processing, vol. 16, no. 4, (2008), pp. 757765 .

[16] International Standard, "Earth - moving machinery - Determination of emission sound pressure level at the operator's position - Stationary test conditions”, ISO 6394: 2008 (E). 
International Journal of Control and Automation Vol.7, No.5 (2014) 